\title{
Concepções e qualidade do sono entre pré-universitários
}

\author{
Conceptions and sleep quality among pre-university students \\ Concepciones y calidad del sueño entre preuniversitarios \\ Juliana Irani Fratucci DE GOBBI ${ }^{\mathbf{1}}$ \\ Ivan da Silva BETETO ${ }^{2}$ \\ Alexandre Prata MARTINS ${ }^{2}$ \\ Havy Alexssander Abrami MEIRELLES ${ }^{2}$ \\ Silke Anna Tereza WEBER $\mathbf{W}^{3}$ \\ Professora do Departamento de Fisiologia, Instituto de Biociências de Botucatu, UNESP, Campus de Botucatu \\ ${ }^{2}$ Graduandos Curso de Medicina, Faculdade de Medicina de Botucatu, UNESP \\ ${ }^{3}$ Professora do Departamento de Cirurgia de Cabeça e Pescoço, Otorrinolaringologia e Oftalmologia, Faculdade de Medicina de Botucatu, UNESP
}

\section{Resumo}

Vários estudos apontam para uma relação entre má qualidade do sono e fatores de risco para doenças crônicas, como por exemplo, depressão e obesidade. Estudantes pré-universitários são mais susceptíveis ao estresse podendo impactar a qualidade do sono. Nosso objetivo foi avaliar conhecimentos prévios do senso comum sobre o sono, bem como a qualidade do sono em estudantes pré-universitários. A pesquisa foi aprovada pelo CEP local (parecer: 2.368.256). Para tanto foram empregados três questionários autoaplicáveis: 1) mitos e verdades sobre o sono, desenvolvido para o estudo, 2) escala de sonolência de Epworth e 3) índice de qualidade do sono de Pittsburgh. Esta pesquisa foi realizada antes das palestras e atividades de divulgação científica de um evento de extensão universitária para difusão sobre neurociêncais. Responderam aos questionários 112 estudantes. Os resultados demonstraram falta de conhecimentos adequados sobre o tema sono, sendo que $58,92 \%$ dos entrevistados entendem que bebidas alcóolicas ajudam no sono, um mito na nossa cultura. Em adição, $70 \%$ desta população apresenta qualidade ruim de sono. Desta forma, nossos resultados apontam que apesar de jovens esta faixa da população já apresenta problemas relacionados à qualidade do sono, e ignoram alguns conhecimentos sobre o tema. Nossa intenção foi despertar, através da divulgação de conhecimentos, a busca por alternativas que melhorem esta qualidade. Durante o evento foram disseminadas ideias sobre a higiene do sono para esta população.

Descritores: Qualidade do Sono; Questionários; Neurociências; Difusão de Conhecimentos.

\section{Abstract}

Several studies point to a relationship between poor sleep quality and risk factors for chronic diseases, such as depression and obesity. Preuniversity students are more susceptible to stress and may impact sleep quality. Our objective was to evaluate previous common-sense knowledge about sleep as well as sleep quality in pre-university students. The survey was approved by the local CEP (\# 2,368,256). Three self-administered questionnaires were used: 1) myths and truths about sleep, developed for this study, 2) Epworth sleepiness scale, and 3) Pittsburgh sleep quality index. This research was conducted prior to the lectures and activities of scientific dissemination of a university extension event for diffusion on neurosciences. 112 students answered the questionnaires. The results showed a lack of adequate knowledge about the topic of sleep, with 58.92\% of those interviewed understand that alcoholic beverages help sleep, a myth in our culture. In addition, $70 \%$ of this population presents poor sleep quality. Thus, our results show that, despite the fact that young people in this population range already present problems related to sleep quality, they ignore some knowledge about the subject. Our intention was to awaken, through the dissemination of knowledge, the search for alternatives that improve this quality. During the event, ideas about sleep hygiene were disseminated to this population.

Descriptors: Sleep Quality, Questionnaires, Neurosciences, Knowledge Diffusion.

\section{Resumen}

Varios estudios apuntan a una relación entre mala calidad del sueño y factores de riesgo para enfermedades crónicas, como por ejemplo, depresión y obesidad. Los estudiantes preuniversitarios son más susceptibles al estrés y pueden afectar la calidad del sueño. Nuestro objetivo fue evaluar conocimientos previos del sentido común sobre el sueño, así como la calidad del sueño en estudiantes preuniversitarios. La encuesta fue aprobada por el CEP local (\# 2.368.256). Para ello se utilizaron tres cuestionarios autoaplicables: 1) mitos y verdades sobre el sueño desarrollado para el estudio, 2) escala de somnolencia de Epworth y 3) índice de calidad del sueño de Pittsburgh. Esta investigación fue realizada antes de las conferencias y actividades de divulgación científica de un evento de extensión universitaria para difusión sobre neurociencias. Respondieron a los cuestionarios 112 estudiantes. Los resultados demostraron falta de conocimientos adecuados sobre el tema del sueño, siendo que el $58,92 \%$ de los entrevistados entienden que las bebidas alcohólicas ayudan en el sueño, un mito en nuestra cultura. Además, el $70 \%$ de esta población presenta una mala calidad de sueño. De esta forma, nuestros resultados apuntan que a pesar de jóvenes esta franja de la población ya presenta problemas relacionados a la calidad del sueño, e ignoran algunos conocimientos sobre el tema. Nuestra intención fue despertar, a través de la divulgación de conocimientos, la búsqueda de alternativas que mejoran esta calidad. Durante el evento se han diseminado ideas sobre la higiene del sueño para esta población.

Descriptores: Calidad del Sueño; Cuestionarios; Neurociencias; Difusión de Conocimiento.

\section{INTRODUÇÃO}

O sono é um estado de alteração da consciência, transitório e reversível, que se alterna com a vigília, estado desperto ${ }^{1,2}$. O sono e a vigília são comportamentos periódicos nítidos, obedecem ao ciclo circadiano; sendo um proceso que envolve complexos mecanismos fisiológicos e comportamentais em vários sistemas e regiões do sistema nervoso central ${ }^{1,2}$ e curiosamente passamos quase um terço de nossas vidas dormindo. Várias funções são atribuídas ao sono sendo que a qualidade desse sono pode alterar nossa saúde. A hipótese mais simples é a de que o sono se destina à recuperação pelo organismo de um possível débito energético estabelecido durante a vigília. Além dessa hipótese, outras funções são atribuídas, especialmente à fase de sono REM (Rapid Eye Movement), tais como: consolidação da memória, regulação da temperatura corporal, um bom equilíbrio neuroimunoendocrinológico, equilíbrio de vários neurotransmissores que afetam o ciclo sono-vigília, bem como manutenção do equilíbrio geral do organismo ${ }^{1,2}$. Pelas razões enumeradas podemos inferir a enorme importância deste comportamento para a manutenção da saúde. A falta de sono adequado é fator de risco para uma série de distúrbios como: a depressão ${ }^{3}$, a doença arterial coronariana ${ }^{4}$, a 
obesidade $^{5}$, o diabetes mellitus ${ }^{6}$ e a hipertensão arterial sistêmica ${ }^{7}$.

Em adição, um fator perturbador para o sono que tem se tornado marcante em nossa sociedade é a exposição constante à luz artificial em atividades interativas com a televisão, internet e principalmente o celular com as várias redes sociais. Esse estilo de vida colabora para os distúrbios do sono, especialmente a insônia e a sonolência diurna excessiva, que se tornaram queixas comuns na população em geral $^{8,9}$. A insônia é o transtorno do sono mais comum, variando em até $50 \%$ na população $^{10}$, seguido da sonolência excessiva diurna $^{11}$. A população de estudantes préuniversitários está mais sujeita a impactos negativos na qualidade do sono gerados por exposição a estresse, conforme sugerem vários estudos ${ }^{12,13}$.

Eventos pautados na divulgação de conhecimentos são bons momentos para acessar conceitos que a população em geral possui sobre um assunto e inserir correções e ideias novas a esse respeito $^{14}$. Um evento de extensão voltado para divulgação de neurociências é a Semana de Conscientização sobre o Cérebro, parte integrante da campanha mundial "Brain Awareness Week". Durante a realização da III Semana em Botucatu-SP, foram divulgadas informações sobre a importância do sono para o sistema nervoso central para alunos do ensino fundamental e médio da cidade de Botucatu. Nesse momento aproveitamos a semana para averiguar através de uma enquete, com afirmativas e a escolha entre verdade ou mito, sobre o quanto os estudantes pré-universitários conheciam sobre o tema. A falta de conhecimento adequado sobre o tema pode refletir na qualidade do sono destes indivíduos. Acessar os conhecimentos prévios desta população foi parte da presente pesquisa.

Ademais, a qualidade do sono pode ser acessada através de questionários. Os mais utilizados e fáceis de serem aplicados são: a escala de sonolência de Epworth (ESE), idealizada com base em observações relacionadas à natureza e à ocorrência da sonolência diurna excessiva ${ }^{15}$; e o índice de qualidade do sono de Pittsburgh (PSQI), questionário já validado para a língua portuguesa, que fornece informações qualitativas e quantitivas sobre a qualidade do sono no último mês ${ }^{16}$. Desta forma, tendo em vista a importância do sono este estudo também visou verificar a qualidade do sono em estudantes pré-universitários tendo por base a resposta para os questionários ESE e PSQI.

\section{MATERIAL E MÉTODO}

\section{- População-alvo}

Este estudo foi realizado durante o evento de extensão sobre divulgação em neurociências, III Semana de Conscientização sobre o Cérebro. Foram entrevistados os estudantes pré-vestibulares $(n=150)$ do extensivo noturno do cursinho Desafio, vinculado a um projeto de extensão da Faculdade de Medicina de Botucatu, UNESP. O projeto foi aprovado pelo Comitê de Ética Local (parecer: 2.368.256). Os participantes foram convidados a assinarem o Termo de Consentimento Livre e Esclarecido (TCLE) autorizando a participação na pesquisa, para os maiores de 18 anos ou responsáveis, bem como o Termo de Assentimento Livre e Esclarecido (TALE) para os menores de 18 anos de idade.

\section{- Questionários Autoaplicáveis}

Após os esclarecimentos sobre as atividades e a pesquisa, foram aplicados 3 questionários:

1. Questionário "Mitos e Verdades do Sono", construído para esta pesquisa. Composto por 12 afirmativas (Tabela 1) e a escolha para cada uma sobre a afimativa ser: verdade, mito ou não sei. Buscamos rastrear lacunas de informações ou distorções promovidas pela mídia e sociedade de forma geral sobre o tema.

2. Escala de Sonolência de Epworth (ESE) que avalia a possibilidade de cochilar em 8 situações cotidianas. Utiliza-se uma escala de 0 a 3 onde, 3 corresponde a grande probabilidade e 0 nenhuma, considerando pontuação > 8 como risco de sonolência diurna. Validada e adaptada para a realidade brasileira $^{17}$.

3. Índice de Qualidade do Sono de Pittsburgh (PSQI) composto de 19 questões agrupadas em sete componentes com pesos distribuídos em uma escala de 0 a 3 , considerando qualidade de sono normal pontuação $<5$. Entre as questões destacam-se a qualidade subjetiva do sono, a latência para o sono, a duração do sono, a eficiência do sono, o uso de medicamentos para dormir e a disfunção diurna. Validado e adaptado para a realidade brasileira $^{18}$.

Após a aplicação dos questionários os estudantes pré-universitários foram convidados a participar de uma palestra em torno de 45 minutos onde foram abordados os temas sobre o que é sono, regiões do encéfalo envolvidos com o processo do sono, suas funções e as respostas corretas sobre a enquete com as explicações para cada afirmativa. Neste momento também foram discutidas e dialogadas os pressupostos em que se baseiam a higiene do sono.

Os questionários foram agrupados e inseridos em tabelas do programa Excel® para estratificação dos dados entre sexo, idade, índice de massa corporal (IMC $\mathrm{kg} / \mathrm{m}^{2}$ ) e pontuação nas escalas, segundo as instruções para cada questão dos questionários. Os resultados estão expressos em porcentagens de vezes que uma dada variável apareceu em relação ao total de respostas. Dos 150 estudantes, 112 responderam 
todo o questionário sobre mitos e verdades, e 101 responderam todas as questões dos questionários ESE e PSQI, bem como assinaram os respectivos termos do comitê de ética.

\section{RESULTADOS}

A tabela 1 mostra os resultados em porcentagem de erro sobre as 12 afirmativas do tema sono para um total de 112 entrevistados (homens $=41$ e mulheres=71). Entre as porcentagens destacamos que $58.92 \%$ consideraram como verdade a frase " $\mathrm{O}$ consumo moderado de bebida alcoólica relaxa e melhora o sono." que na realidade trata-se de um mito. $\mathrm{O}$ item 10 da tabela 1 , sobre o uso do aparelho celular, aponta que um terço dos estudantes pensa que não há problemas em levar o celular para a cama, sendo que este hábito atrapalha o sono. $\mathrm{O}$ item $11 \mathrm{da}$ tabela 1 também merece destaque, para a afirmativa: "Colocar pijama para dormir ajuda na qualidade do sono", $60,71 \%$ dos participantes a apontaram como mito, sendo que é uma afirmativa verdadeira. A enquete evidencia a necessidade de maior conhecimento sobre o assunto sono.

Tabela 1. Porcentagem de respostas erradas sobre enquete de mitos e verdades relacionados à conceitos à respeito do sono $\mathrm{e}$ funcionamento do cérebro

\begin{tabular}{|c|c|c|}
\hline $\begin{array}{l}\text { Dados } \\
\text { Gerais }\end{array}$ & $\begin{array}{l}\text { Porcentagem de } \\
\text { erro }\end{array}$ & Questões \\
\hline \multirow[b]{4}{*}{$\begin{array}{l}\text { Número de } \\
\text { entrevistados } \\
=119\end{array}$} & $49,10 \%$ & Dormir faz crescer. (verdade) \\
\hline & $41,96 \%$ & $\begin{array}{l}\text { 2. Roncar é natural e não faz mal à } \\
\text { saúde. (mito) }\end{array}$ \\
\hline & $58,92 \%$ & $\begin{array}{l}\text { 3. O consumo moderado de bebida } \\
\text { alcoólica relaxa e melhora o sono. } \\
\text { (mito) }\end{array}$ \\
\hline & $44,64 \%$ & $\begin{array}{l}\text { 4. O importante é dormir de seis a oito } \\
\text { horas, mesmo que sejam dividas ao } \\
\text { longo do dia. (mito) }\end{array}$ \\
\hline \multirow{8}{*}{$\begin{array}{l}\text { Homens }=41 \\
\text { Mulheres }=71\end{array}$} & $28,57 \%$ & $\begin{array}{l}\text { 5. Posso dormir bastante para poupar o } \\
\text { sono que perderei. (mito) }\end{array}$ \\
\hline & $26,78 \%$ & $\begin{array}{l}\text { 6. Cochilar até meia hora à tarde faz bem. } \\
\text { (verdade) }\end{array}$ \\
\hline & $33,92 \%$ & $\begin{array}{l}\text { 7. } \begin{array}{l}\text { Dormir e acordar mais ou menos no } \\
\text { mesmo horário faz bem. (verdade) }\end{array} \\
\end{array}$ \\
\hline & $41,07 \%$ & $\begin{array}{l}\text { 8. Posso repor o sono perdido no fim de } \\
\text { semana. (mito) }\end{array}$ \\
\hline & $65,17 \%$ & 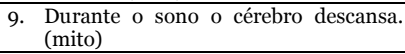 \\
\hline & $25,89 \%$ & $\begin{array}{l}\text { 10. Não tem problema navegar no celular } \\
\text { quando estou deitado para dormir. } \\
\text { (mito) }\end{array}$ \\
\hline & $60,71 \%$ & $\begin{array}{l}\text { 11. Colocar pijama para dormir ajuda na } \\
\text { qualidade do sono. (verdade) }\end{array}$ \\
\hline & $22,32 \%$ & $\begin{array}{l}\text { 12. Dormir pode fazer mal, me deixando } \\
\text { preguiçoso. (mito) }\end{array}$ \\
\hline
\end{tabular}

Na tabela 1, as respostas estão expressas em porcentagem de erros para cada afirmativa. Entre parênteses ao final das frases está a resposta correta, se uma verdade ou um mito.

Os resultados dos questionários de sonolência diruna ESE, como também os dados demográficos são apresentados na tabela 2. Os resultados estão expressos como mediana, sendo a idade de 18 anos a prevalente. $\mathrm{O}$ valor mediano de IMC foi de $22 \mathrm{~kg} / \mathrm{m}^{2}$ estando dentro da normalidade $\left(18,50-24,99 \mathrm{~kg} / \mathrm{m}^{2}\right)$, evidenciando que a população estudada se apresenta eutrófica. Também foi perguntado sobre a prática regular de atividades físicas, sendo que $41,53 \%$ das mulheres e $69,44 \%$ dos homens praticam alguma atividade física como caminhada, andar de bicleta ou jogar futebol. Para o questionário ESE a mediana foi uma pontuação de 8 para os homens e 10 para as mulheres. Na tabela 2 temos as porcentagens entre a normalidade, a média e anormalidade para sonolência diurna. De acordo com a pontuação, $48,57 \%$ dos homens e $66,15 \%$ das mulheres apresentam pontuação acima de 8 , apontando para uma alta sonolência diurna, o que pode indicar algum distúrbio do sono.

Tabela 2. Panorama geral sobre a população de estudantes e pontuação na escala ESSE

\begin{tabular}{|c|c|c|c|c|}
\hline Participantes & Idade & IMC & $E S E$ & \% da Pontuação \\
\hline \multirow{3}{*}{$\begin{array}{l}\text { homens } \\
(\mathrm{n}=36)\end{array}$} & \multirow{3}{*}{$18(17-20)$} & \multirow{3}{*}{$22(21-25)$} & \multirow{3}{*}{$8(6-11)$} & Normal- 40,00\% \\
\hline & & & & Média- $11,43 \%$ \\
\hline & & & & Anormal- 48,57\% \\
\hline \multirow{3}{*}{$\begin{array}{l}\text { mulheres } \\
(\mathrm{n}=65)\end{array}$} & \multirow{3}{*}{$18(17-19)$} & \multirow{3}{*}{$22(19-25)$} & \multirow{3}{*}{$10(8-13)$} & Normal- $10,77 \%$ \\
\hline & & & & Média- 23,08\% \\
\hline & & & & Anormal- 66,15\% \\
\hline
\end{tabular}

Na tabela 2, os resultados estão expressos como mediana, primeiro e terceiro interquartis. IMC $\left(\mathrm{kg} / \mathrm{m}^{2}\right)=$ índice de massa corporal; ESE = escala de sonolência de Epworth. De acordo com a pontuação da ESE: 1 - 6 pontos: sono normal; 7 - 8 pontos: média de sonolência; 9 - 24 pontos: sonolência anormal.

Com respeito a qualidade do sono a tabela 3 mostra a porcentagem da população estudada com qualidade boa, ruim ou presença de distúrbio, bem como a latência, duração e eficiência do sono para cada tipo de qualidade. A transição da vigília para o sono, latência, é maior para as mulheres com distúrbio, sendo que a diferença de mediana entre homens e mulheres chega a 25 minutos de latência a mais para as mulheres. A duração de horas dormidas cai acentuadamente, quase para a metade do tempo, evidenciando a presença de distúrbio. As mulheres também apresentam queixa de ansiedade maior (homens $8,33 \%$ versus mulheres 23,07\%). Com relação ao uso de alguma medicação para induzir o sono $13,88 \%$ dos homens e $18,46 \%$ das mulheres relatam uso de alguma medicação para induzir o sono.

Tabela 3. Resultados relacionados à qualidade do sono segundo o questionário PSQI

\begin{tabular}{l|lllll}
\hline Participantes & PSQI & $\begin{array}{l}\text { Latência } \\
\text { (min) }\end{array}$ & $\begin{array}{l}\text { Duração } \\
\text { (horas) }\end{array}$ & Eficiência & $\begin{array}{l}\text { Queixa } \\
\text { ansiedade }\end{array}$ \\
\hline \multirow{4}{*}{$\begin{array}{l}\text { Homens } \\
(\mathrm{n}=36)\end{array}$} & $\begin{array}{l}\text { Boa- } \\
36,11 \%\end{array}$ & $10(5-15)$ & $8(7-9)$ & $93,75 \%$ & \\
\cline { 2 - 5 } & $\begin{array}{l}\text { Ruim- } \\
58,33 \%\end{array}$ & $20(10-30)$ & $7(6-8)$ & $93,57 \%$ & \\
\cline { 2 - 5 } & $\begin{array}{l}\text { Distúrbio } \\
-5,55 \%\end{array}$ & $65(53-78)$ & $4,3(4-5)$ & $87,22 \%$ & \\
\hline \multirow{4}{*}{$\begin{array}{l}\text { Mulheres } \\
(\mathrm{n}=65)\end{array}$} & $\begin{array}{l}\text { Boa- } \\
15,38 \%\end{array}$ & $10(10-14)$ & $8(7-9)$ & $94,56 \%$ & \\
\cline { 2 - 5 } & $\begin{array}{l}\text { Ruim- } \\
76,92 \%\end{array}$ & $22(15-30)$ & $7(6-8)$ & $91,90 \%$ \\
\cline { 2 - 5 } & $\begin{array}{l}\text { Distúrbio } \\
-7,69 \%\end{array}$ & $90(60-90)$ & $4(3-7)$ & $86,16 \%$ & \\
\hline
\end{tabular}

Os resultados apresentados na tabela 3 estão em porcentagens do total para cada pontuação, e como mediana, primeiro e terceiro interquartis. PSQI = índice da qualidade de sono de Pittsburgh. Pontuação global do PSQI, de acordo com a soma dos 7 componentes do questionário: boa: 0-4, ruim: 
5-10 e distúrbio > 10. Latência - tempo estimado para transição vigília-sono; duração estimada do tempo de sono; eficiência: considera uma porcentagem entre duração do sono e latência.

\section{DISCUSSÃO}

Nossos resultados mostraram que a população de estudantes pré-universitários apresenta desconhecimento sobre o funcionamento do cérebro durante o sono bem como aspectos relacionados ao sono. Os resultados também apontaram para uma qualidade ruim de sono na maioria desta população, evidenciando a necessidade de maior atenção e intervenção sobre como melhorar esta qualidade com vistas a promoção da saúde em longo prazo.

Segundo os nossos resultados da enquete fica evidente que ações de divulgação de conhecimentos para a população são importantes para ajudar a diminuir a mistificação sobre alguns aspectos relacionados à neurociências, inclusive numa população que possui acesso a informações e que almeja uma posição para o terceiro grau. A disseminação de conhecimentos é importante para colaborar na promoção da saúde, através da conscientização para mudança de hábitos apontados como mais saudáveis. $O$ tratamento não farmacológico para distúrbios do sono ganha forma com terapias comportamentais e ações ambientais relacionadas ao ritual do ato de dormir, incluindo a higiene do sono ${ }^{19,20}$. Um dos pressupostos da higiene do sono é evitar uso de álcool ou estimulantes perto da hora do sono. Com relação ao álcool existe um mito no senso comum que a ingestão de bebida alcóolica poderia colaborar no sono, e esse senso foi revelado em nossa enquete que apontou que 58,92\% dos estudantes concordaram que o consumo de bebida alcóolica melhora o sono. $\mathrm{Na}$ realidade $\mathrm{o}$ álcool é um sedativo e reduz a latência para o sono, porém seu uso diminui a porcentagem do sono REM e consequentemente, a sua qualidade ${ }^{21,22}$. Esta porcentagem alta de confusão conclama mais campanhas de divulgação científica para disseminação de conceitos corretos em nossa sociedade.

Similarmente sobre hábitos relacionados ao sono, um aspecto simples e fácil de ser corrigido é a colocação de um pijama para dormir. Isto ajuda na criação de uma associação mental pijama-dormir que contribui para melhorar a latência e qualidade do sono. Em adição, é um hábito higiênico dormir com uma roupa que não foi usada para outros afazeres. Nesta afirmativa $60,71 \%$ dos estudantes encaram isto como um mito, mais uma vez evidenciando a importância da disseminação de informações corretas para a sociedade, o que pode gerar a mudança de hábitos e a promoção da saúde. Outro erro muito comum, que atingiu $65,17 \%$ dos entrevistados, é o fato de crerem que o cérebro descansa enquanto dormimos, como se existisse um apagão cerebral, e novamente a informação científica colabora na desmistificação deste fato. $\mathrm{O}$ padrão de atividade cerebral muda, mas ele não cessa ${ }^{1}$.

$\mathrm{Na}$ nossa investigação, a maioria dos participantes apresenta uma qualidade ruim do sono, apresentando uma latência de até 30 minutos para o sono, tanto homens quanto mulheres. A quantidade de horas de sono para a maioria apresentou uma mediana de 7 horas por noite, estando dentro do padrão. Por outro lado, dentro desta maioria, há uma fragmentação grande do sono (mulheres $32,00 \%$ e homens 33,33\%) com despertares de até 3 vezes durante a noite. Embora a população investigada seja composta por jovens eutróficos, sem comorbidades e com bons hábitos de atividades físicas regulares, eles exibem na maioria uma qualidade ruim de sono. Nas mulheres a qualidade ruim de sono em $76,92 \%$ na escala PQSI está em coerência com a pontuação ESE que mostrou sonolência diurna excessiva em $66,15 \%$ delas. É interessante ressaltar que quando analisada a subjetividade do sono $50,00 \%$ das mulheres e $52,38 \%$ dos homens classificam como bom o próprio sono, talvez isto aconteça por serem ainda jovens e saudáveis.

Existem alguns relatos sobre a qualidade de sono em estudantes universitários ${ }^{23-25}$, porém na população de estudantes pré-universitários esta informação é escassa. Alguns estudos ${ }^{26,27}$ relacionam a má qualidade do sono ao crescente uso de tecnologias (celular, computador, televisão entre outros), com exposição à luz azul, fragmentação de sono e manutenção de atividade intensa cerebral para processar todas as imagens/informações. Este estado de excitação pode ser inferido na queixa dos adolescentes de não conseguirem dormir por excesso de pensamentos. Neste estudo, a investigação de uso excessivo de tecnologia não foi o objetivo, mas o fato de pelo menos um terço deles não considerarem a navegação durante a noite como um problema, mostra que não há restrição de uso.

Um aspecto interessante para préuniversitários é o aumento da ansiedade pelas incertezas que enfrentam, a ansiedade apareceu em nosso estudo como queixa para iniciar ou manter o sono, sendo maior em mulheres em relação aos homens, em coerência com outros estudos em população pré-universitária ${ }^{13}$. Há diversos estudos relacionando depressão/ansiedade com distúrbios do sono, sendo que a adoção de uma boa higiene de sono trazendo resultados benéficos. Em nosso estudo, o uso elevado de medicações que ajudam a induzir o sono mostra que há pouco conhecimento da população geral sobre medidas não medicamentosas para melhorar a qualidade do sono. Um aspecto que vale ressaltar é que nossa população estudada é composta de jovens que além da atividade escolar ainda frequentam o cursinho pré-vestibular à noite. 
Esta "jornada dupla" e o estresse causado pela expectativa da prova de vestibular certamente contribuem para a ansiedade e para a privação de sono. Assim, neste momento tão vulnerável há uma necessidade maior de divulgação de informação sobre hábitos ruins de sono, o risco do uso excessivo de tecnologias e do benefício das medidas de higiene do sono.

\section{CONCLUSÃO}

Com base na metodologia empregada e
resultados obtidos observou-se falta de
conhecimentos adequados sobre o tema sono e o
cérebro, apontando para uma qualidade ruim de sono
em estudantes pré-universitários. A intensificação de
disseminação de conhecimentos a este respeito pode
colaborar para uma melhor qualidade do sono, bem
como a promoção da saúde.

\section{REFERÊNCIAS}

1. Bear MF, Connors BW, Paradiso M. A. Neurociências: desvendando o sistema nervoso. 3. ed. Porto Alegre: Artmed, 2007. 586-616p.

2. Tufik S, Andersen ML, Pinto Jr LR. Sono e sonhos, In: Lent R (ed). Neurociência da mente e do comportamento. Rio de Janeiro: Guanabara Koogan; 2008.

3. Riemann D, Voderholzer U. Primary insomnia: a risk factor to develop depression? J Affect Disord.2003;76(1-3):255-59

4. Greenland P, Knoll MD, Stamler J, Neaton JD, Dyer AR, Garside DB et al. Major risk factors as antecedents of fatal and nonfatal coronary heart disease events. JAMA. 2003;290(7):891-97.

5. Watanabe M, Kikuchi H, Tanaka K, Takahashi M. Association of short sleep duration with weight gain and obesity at 1-year follow-up: a large-scale prospective study. Sleep. 2010;33(2):161-67.

6. Spiegel K, Knutson K, Leproult R, Tasali E, Van Cauter E. Sleep loss: a novel risk factor for insulin resistance and Type 2 diabetes. J Appl Physiol. 1985;99(5):2008-19.

7. Vgontzas AN, Liao D, Bixler EO, Chrousos GP, Vela-Bueno A. Insomnia with objective short sleep duration is associated with a high risk for hypertension. Sleep. 2009;32(4):491-97.

8. Fossum IN, Nordnes LT, Storemark SS, Bjorvatn B, Pallesen S. The association between use of electronic media in bed before going to sleep and insomnia symptoms, daytime sleepiness, morningness, and chronotype. Behav Sleep Med. 2014;12(5):343-57.

9. Freitas CCM, Gozzoli ALDM, Konno JN, Fuess VLR. Relação entre uso do telefone celular antes de dormir, qualidade do sono e sonolência diurna. Rev Med (São Paulo). 2017;96(1):14-20.

10.Poyares D, Tufik S. I Consenso Brasileiro de Insônia: introdução. Hypnos. 2003:5-45.
11.Guilleminault C, Brooks SN. Exccessive daytime sleepness: a challenge for the practising neurologist. Brain. 2001;124(8):1482-91.

12.Peruzzo AS, Cattani BC, Guimarães ER, Boechat L deC, Argimon II deL, Scarparo HBK. Estresse e vestibular como desencadeadores de somatizações em adolescentes e adultos jovens. Psicol Argum. 2008;26(55):319-27.

13.Rodrigues DG, Pelisoli C. Ansiedade em vestibulandos: um estudo exploratório. Rev psiquiatr clín. 2008;35(5):171-77.

14.Fragel-Madeira L, Aranha G. Divulgação e alfabetização científica: o papel do pesquisador na difusão do conhecimento científico. In: Aranha G, Sholl-Franco A (org). Caminhos da Neuroeducação. 2.ed: Rio de Janeiro: Ciências e Cognição. p.119-28; 2012.

15.Johns MW. A new method for measuring daytime sleepiness: the Epworth sleepiness scale. Sleep. 1991;14(6):540-45.

16.Buysse DJ, Reynolds CF 3rd, Monk TH, Berman SR, Kupfer DJ. The Pittsburgh Sleep Quality Index: a new instrument for psychiatric practice and research. Psychiatry Res.1989;28(2):193-213.

17.Bertolazi AN, Fagondes SC, Hoff LS, Pedro VD, Mena Barreto SS, Johns MW et al. Validação da escala de sonolência de Epworth em português para uso no Brasil. J Bras Pneumol. 2009; 35(9):877-83.

18. Bertolazi AN, Fagondes SC, Hoff LS, Dartora EG, Miozzo IC, de Barba ME. Validation of the Brazilian Portuguese version of the Pittsburgh Sleep Quality Index. Sleep Med. 2011;12(1):70-5.

19. Morgenthaler T, Kramer M, Alessi C, Friedman $\mathrm{L}$, Boehlecke B, Brown $\mathrm{T}$ et al. Practice parameters for the psychological and behavioral treatment of insomnia: An update. an American academy of sleep medicine report. Sleep. 2011; 29(11):1415-19.

20.Passos GS, Tufik S, Santana MG, Poyares D, Mello MT. Tratamento não farmacológico para a insônia crônica. Rev Bras Psiquiatr. 2007;29(3):279-82.

21.Ebrahim IO, Shapiro CM, Williams AJ, Fenwick PB. Alcohol and sleep I: effects on normal sleep. Alcohol Clin Exp Res. 2013;37(4):539-49.

22.Irish LA, Kline CE, Gunn HE, Buysse DJ, Hall $\mathrm{MH}$. et al. The role of sleep hygiene in promoting public health: A review of empirical evidence. Sleep Med Rev. 2015;22:23-36.

23. Carvalho TMCS, Silva Junior II, Siqueira PPS, Almeida JO, Soares AF, Lima AMJ. Qualidade do sono e sonolência diurna entre estudantes universitários de diferentes áreas. Rev Neurocienc. 2013;21(3):383-87.

24.Corrêa CC, Oliveira FK, Pizamiglio DS, Ortolan EVP, Weber SAT. Qualidade de sono em 
estudantes de medicina: comparação das diferentes fases do curso. J Bras Pneumol. 2017;43(4):285-89.

25.Vasconcelos HCA, Fragoso LVC, Marinho NBP, Araújo MFM, Freitas RWJF, Zanetti ML et al. Correlação entre indicadores antropométricos e a qualidade do sono universitários brasileiros. Rev Esc Enferm USP. 2013;47(4):852-59.

26. Ferreira C, Ferreira H, Vieira MJ, Costeira M, Branco L, Dias A et al. Epidemiologia do uso de internet numa população adolescente e sua relação com hábitos de sono. Acta Med Port. 2017; 30(7-8):524-33.

27.Peter ID, Adamu H, Asani MO, Aliyu I, Sabo UA, Umar UI. Sleep pattern and sleep hygiene practices among nigerian schooling adolescents. Indian J Psychol Med. 2017;39(4):407-12.

\section{CONFLITO DE INTERESSES}

Os autores declaram não haver conflitos de interesse.

AUTOR PARA CORRESPONDENCIA

Juliana Irani Fratucci de Gobbi

juliana.gobbi@unesp.br

Submetido em 23/01/2019

Aceito em 18/03/2019 